\title{
STABILITY OF POISEUILLE FLOW IN PIPES, ANNULI, AND CHANNELS*
}

\author{
BY \\ D. D. JOSEPH AND S. CARMI \\ University of Minnesota
}

Summary. The value of $R=180$ which has been given by Orr [1] as a limit for sure stability of Hagen-Poiseuille flow is incorrect. A lower value, $R=82.88$, can be associated with an eigenfunction possessing a first mode azimuthal variation $(N=1)$ and no streamwise variation. This eigenfunction is obtained as an exact solution of the appropriate Euler equation. A yet lower value, $R=81.49$, is associated with a spiral mode with $N=1$ and wave number $\alpha \approx 1$. Corresponding results are obtained for Poiseuille flow between cylinders. For all but the very smallest radius ratios the smallest eigenvalue of Euler's equation is assumed for the purely azimuthal disturbance. The mode shape for the pipe flow is consistent with the experimental situation as it is now understood [2], though the stability limit is much smaller than the experimental value $(R \approx 2100)$. For the annulus, the variation of the energy limit with the radius ratio (from pipe flow to channel flow) is consistent with the experimentally observed stability limit. All the results of the energy analysis hold equally if the pipe is in rigid rotation about its axis. If the rotation is "fast" linear and energy results nearly coincide.

Consider the arbitrary motion of a viscous fluid governed by the Navier-Stokes equations. It is known that if the Reynolds number ( $\mathrm{Re}$ ) of this motion satisfies the inequality

$$
\operatorname{Re}<R
$$

where

$$
\begin{gathered}
\frac{1}{R}=\operatorname{Max}_{\nabla} \frac{-\int_{v} \mathbf{v} \cdot \mathbf{E} \cdot \mathbf{v d x}}{\int_{v} \nabla \mathbf{v}: \nabla \mathbf{v d x}}, \\
\mathbf{v}=0 \quad(\mathbf{x} \in \partial v), \\
\operatorname{div} \mathbf{v}=0 \quad(\mathbf{x} \in v),
\end{gathered}
$$

then the motion is asymptotically stable in the mean. Here $v$ is a bounded region and

$$
\begin{aligned}
m E_{i j} & =\frac{1}{2}\left(\frac{\partial V_{i}}{\partial X_{i}}+\frac{\partial V_{i}}{\partial X_{i}}\right), \\
\operatorname{Re} & =m d^{2} / \nu,
\end{aligned}
$$

where $m$ is a constant, $\nu$ is the kinematic viscosity, $d$ is the unit of length, $\mathrm{V}$ is the velocity of the basic motion, and $\mathbf{v}$ is the difference in the velocity of the basic and disturbed motions. The region $v$ may also be taken as unbounded in directions for which $v$ may

${ }^{*}$ Received October 2, 1967; revised version received August 16, 1968. Prepared under NSF Grant GK1838. 
be taken as spatially periodic or nonperiodic but square integrable with square integrable first derivatives.

Eq. (2) is a consequence of manipulations starting from energy identities of Reynolds [3]. The variational problem implied by (2) was given explicitly by Orr [1] who solved the associated Euler equations for the simpler rectilinear viscous motions. A general formulation of this problem was given for the first time by Serrin [4] and the proof of the sufficiency of condition (1) is to be found in [5]. ${ }^{1}$

The Euler equations for (2), (3), and (4) are

$$
\begin{gathered}
\rho \mathbf{E} \cdot \mathbf{v}=\Delta \mathbf{v}-\nabla p, \quad \operatorname{div} \mathbf{v}=0, \\
\mathbf{v}=\left.0\right|_{\partial v},
\end{gathered}
$$

where the quantity $p$ enters as a Lagrange multiplier through the divergence constraint. The value $R$ of (1) is found as the smallest positive eigenvalue $\rho$ of the systems (5) and (6).

Orr [1] obtained that version of (5) and (6) which governs the rectilinear motion of a viscous fluid between plane walls and in tubes, that is, for Poiseuille and Couette flows. $\mathrm{He}$ has also given certain two-dimensional solutions and values $\rho$ for these problems. These values have been authoritatively cited as stability limits for sixty years but are largely incorrect.

The essential defect in Orr's analysis is an unfounded belief in the destabilizing effects of streamwise disturbances and the suppression of certain transverse modes. Ironically, the uncritical acceptance of this fallacious argument may well have been supported by its correctness relative to the Orr-Sommerfeld problem (Squires theorem).

For plane-Couette flow (Joseph [5]) and Hagen-Poiseuille flow lower values of $\rho$ than those given by Orr can be associated with modes in which streamwise variations are suppressed. For Hagen-Poiseuille flow we here obtain the value

$$
\rho=81.49,
$$

for a disturbance with a dimensionless wave number $\alpha=1.07$ and $N=1$. We prove that $R<82.88$ ( $\alpha=0$ and $N=1$ ) and establish an analytical basis (but not a proof) for the numerical result

$$
R=81.49
$$

The stability of Poiseuille flow in the annulus is taken up in Sec. 7. We first develop a detailed analysis for the pipe flow.

1. The maximum problem and the Euler equations. Consider the rectilinear motion of a viscous fluid down a pipe of uniform circular cross section of radius $r_{0}$. The solution (cylindrical coordinates)

$$
\mathrm{V}=(W, V, U)=\left(0,0, U_{M}\left[1-\left(r^{*} / r_{0}\right)^{2}\right]\right)
$$

is readily obtained from the Navier-Stokes equations. The deformation matrix for this motion is given by

$$
\mathbf{E}=-\frac{U_{M} r^{*}}{m r_{0}^{2}}\left(\begin{array}{lll}
0 & 0 & 1 \\
0 & 0 & 0 \\
1 & 0 & 0
\end{array}\right)
$$

${ }^{1}$ The theorem is a joint result of Joseph and Serrin. 
Set

$$
m=U_{M} / r_{0}, \quad d=r_{0}, \quad r=r^{*} / r_{0}, \quad \operatorname{Re}=U_{M} r_{0} / \nu
$$

and

$$
\rho E \cdot \mathrm{v}=-\rho r\left(v_{z}, 0, v_{r}\right)
$$

where

$$
\mathbf{v}=\left(v_{r}, v_{\theta}, v_{z}\right) \text { and } \mathbf{r}=(r, \theta, z)
$$

The maximum problem (2) makes sense in the unbounded domain when the solutions are single-valued in $\theta$ and (i) periodic in $z$ or (ii) not periodic in $z$, but are square integrable with square integrable derivatives on $z \in(-\infty, \infty)$. The periodic solutions can be represented by normal modes

$$
\mathbf{v}(r, \theta, z) \rightarrow \mathfrak{u}(r, \alpha, N) e^{i(\alpha z+N \theta)}
$$

which are single-valued in $\theta$ when $N$ is an integer. The nonperiodic solutions can be represented by Fourier transforms

$$
\mathbf{v}=\sum_{N=-\infty}^{\infty} \int_{-\infty}^{\infty} \mathbf{u}(r, \alpha, N) e^{i(N \theta+\alpha z)} d \alpha
$$

where

$$
\mathbf{u}=1 / 4 \pi^{2} \int_{-\infty}^{\infty} d z \int_{0}^{2 \pi} \mathbf{v}(r, \theta, z) e^{i(N \theta+\alpha z)} d \theta
$$

Similar expressions are used for $p(r, \theta, z)$.

Ordinary differential equations for $\mathbf{u}$ (of either class) may be obtained from (5) in the routine way. It is, however, instructive to derive these equations directly from (2) without introducing the multiplier $p$. First write the real-valued integrands of (2) as combinations of complex functions. Then, relative to $\nabla$ of class (12a) or (12b) we seek

$$
1 / R=\operatorname{Sup}(I(\mathbf{v}) / D(\mathbf{v}))
$$

where

$$
\begin{aligned}
& I(\mathrm{v})=\int_{-\infty}^{\infty} d z \int_{0}^{2 \pi} d \theta \int_{0}^{1} r^{2}\left(v_{r} v_{z}^{*}+v_{r}^{*} v_{z}\right) d r \\
& D(\mathrm{v})=\int_{-\infty}^{\infty} d z \int_{0}^{2 \pi} d \theta \int_{0}^{1} r \nabla \mathrm{v}: \nabla \mathrm{v}^{*} d r
\end{aligned}
$$

and

$$
\begin{aligned}
\nabla \mathrm{v}: \nabla \mathrm{v}^{*}=\left|\frac{\partial v_{r}}{\partial r}\right|^{2}+\frac{1}{r^{2}}\left|\frac{\partial v_{r}}{\partial \theta}\right|^{2}+\left|\frac{\partial v_{r}}{\partial z}\right|^{2} & +\left|\frac{\partial v_{\theta}}{\partial r}\right|^{2}+\frac{1}{r^{2}}\left|\frac{\partial v_{\theta}}{\partial \theta}\right|^{2}+\left|\frac{\partial v_{\theta}}{\partial z}\right|^{2} \\
& +\left|\frac{\partial v_{z}}{\partial r}\right|^{2}+\frac{1}{r^{2}}\left|\frac{\partial v_{z}}{\partial \theta}\right|^{2}+\left|\frac{\partial v_{z}}{\partial z}\right|^{2}+\frac{\left|v_{r}\right|^{2}+\left|v_{\theta}\right|^{2}}{r^{2}} \\
& +\frac{1}{r^{2}}\left(v_{r} \frac{\partial v_{\theta}^{*}}{\partial \theta}+v_{r}^{*} \frac{\partial v_{\theta}}{\partial \theta}-v_{\theta} \frac{\partial v_{r}^{*}}{\partial \theta}-v_{\theta}^{*} \frac{\partial v_{r}}{\partial \theta}\right)
\end{aligned}
$$


Problem (13a) may be reduced, relative to either (12a) or (12b) to considerations involving the functional $I_{\alpha N}(\mathrm{u}) / D_{\alpha N}(\mathrm{u})$ where

$$
\begin{gathered}
I_{\alpha N}(\mathfrak{u})=\int_{0}^{1} r^{2}\left(w u^{*}+w^{*} u\right) d r \equiv\left\langle r w u^{*}\right\rangle+\left\langle r w^{*} u\right\rangle, \\
D_{\alpha N}(\mathfrak{u})=-\left\langle w, \&_{N} W^{*}\right\rangle-\left\langle v, \mathfrak{L}_{N} v^{*}\right\rangle-\left\langle u, L_{N} u^{*}\right\rangle-2 i N\left(\left\langle v^{*} / r, w / r\right\rangle-\left\langle w^{*} / r, v / r\right\rangle\right), \\
=\left\langle|D w|^{2}+|D v|^{2}+|D u|^{2}+\alpha^{2}\left(|w|^{2}+|v|^{2}+|u|^{2}\right)\right\rangle \\
+N^{2}\left\langle\left|\frac{u}{r}\right|^{2}\right\rangle+\left\langle\left|\frac{N w+i v}{r^{2}}\right|^{2}+\left|\frac{N v-i w}{r^{2}}\right|^{2}\right\rangle,
\end{gathered}
$$

with $\mathfrak{L}_{N}=(1 / r) D(r D)-\left(N^{2}+1\right) / r^{2}-\alpha^{2}, \mathfrak{L}_{N}+\left(1 / r^{2}\right)=L_{N}=L, D=d / d r$, for solenoidal $\mathrm{v}$ satisfying

$$
(1 / r) D(r w)+i N v / r+i \alpha u=0 .
$$

We want to show that for functions of the class (12a) or (12b)

$$
\frac{1}{R}=\operatorname{Max}_{\alpha, N}\left\{\operatorname{Max} \frac{I_{\alpha N}(\mathfrak{u})}{D_{\alpha N}(\mathfrak{u})}\right\}=\operatorname{Max}_{\alpha, N} \rho^{-1}(\alpha, N) .
$$

Consider functions of the class (12b) and let $\mathfrak{u}^{\prime}=(u, w)$. Then by elimination of $v$ in $\mathbf{u}$ through (17) we have

$$
I_{\alpha N}(\mathfrak{u}) / D_{\alpha N}(\mathfrak{u})=\mathscr{g}_{\alpha N}\left(\mathbf{u}^{\prime}\right) / D_{\alpha N}\left(\mathbf{u}^{\prime}\right),
$$

the latter quotient being without side constraints. ${ }^{2}$ Now consider the space of completion elements $\mathfrak{u}^{\prime} \in H$ under the norm $\left(D_{\alpha N}\left(\mathbf{u}^{\prime}\right)^{1 / 2}\right.$ and define the scalar products $\left(\mathbf{u}^{\prime}, \mathbf{u}^{\prime \prime}\right)$ and $\left[\mathbf{u}^{\prime}, \mathbf{u}^{\prime \prime}\right]$ corresponding to the quadratic forms

$$
\left(\mathbf{u}^{\prime}, \mathbf{u}^{\prime}\right)=\mathscr{I}_{\alpha N}\left(\mathbf{u}^{\prime}\right), \quad\left[\mathbf{u}^{\prime}, \mathbf{u}^{\prime}\right]=D_{\alpha N}\left(\mathbf{u}^{\prime}\right) .
$$

For fixed $\mathfrak{u}^{\prime \prime} \in H$ (in fact, for $\left.\mathfrak{u}^{\prime \prime} \in L_{2}\right)\left(\mathfrak{u}^{\prime}, \mathfrak{u}^{\prime \prime}\right)=f_{\mathfrak{u}} \cdot\left(\mathfrak{u}^{\prime}\right)$ is a linear functional on $H$ and by Riesz's Theorem there exists a linear operator A such that $\left(\mathbf{u}^{\prime}, \mathbf{u}^{\prime \prime}\right)=\left[\mathbf{u}^{\prime \prime}, \mathbf{A} \cdot \mathbf{u}^{\prime}\right]$ for every $\mathfrak{u}^{\prime \prime} \in H$. The operator $\mathrm{A}$ is selfadjoint because $\mathscr{I}_{\alpha N}$ is hermitian symmetric. The operator $\mathbf{A}$ is compact because it is the composition of a compact embedding operator $H \rightarrow L_{2}$ and the bounded operator A carrying $L_{2} \rightarrow H$, which arises from Riesz's Theorem. It is known (Riesz-Nagy, [6]) that $\mathbf{A}$ has at least one eigenvalue of, at most, finite multiplicity and the eigenfunctions of $\mathbf{A}$ are complete on the invariant subspace of elements $\mathbf{u}^{\prime} \in H$ such that $\left[\mathbf{u}^{\prime}, \mathbf{A} \cdot \mathbf{u}^{\prime}\right] \neq 0$. All of the nonzero eigenvalues of $\mathbf{A}$ may be obtained from extremum problems. (A is not a positive operator because $\mathfrak{g}_{\alpha N}\left(\mathbf{u}^{\prime}\right)$ is an indefinite quadratic form.) In particular, if there exists at least one $\mathbf{u}^{\prime} \in H$ (which we verify, a posteriori) such that $\mathscr{I}_{\alpha N}\left(\mathfrak{u}^{\prime}\right)>0$ then the largest eigenvalue of $\mathbf{A}$ is positive and

$$
0<\rho^{-1}(\alpha, N)=\operatorname{Max}_{\mathbf{u}^{\prime} \in H} g_{\alpha N}\left(\mathbf{u}^{\prime}\right), \quad D_{\alpha N}\left(\mathbf{u}^{\prime}\right)=1 .
$$

This characterization, and the existence of maximizing element $\mathfrak{u}^{\prime}$ is guaranteed so long as the elimination of $v$ (or $u$ for $N=0$ from $u$ through (17) can be carried through, that is, for each pair of finite values $(\alpha, N) \neq(0,0)$. Lemmas 1 and 2, to be proved below,

${ }^{2}$ When $N=0$ the smallest values of $D_{\alpha N}$ are necessarily found among the $\mathbf{u}$ such that $v \equiv 0$. Then we use (17) to eliminate $\mathbf{u}$. 
guarantee that the largest of the values $\rho^{-1}(\alpha, N)$ over $-\infty \leq \alpha, N<\infty$ are found for finite $(\alpha, N) \neq(0,0)$.

For any $\nabla$ of the nonperiodic class we have from $(12 b)$ that

$$
\begin{aligned}
& \frac{I(\mathbf{v})}{D(\mathbf{v})}=\left\{\sum_{N=-\infty}^{\infty} \int_{-\infty}^{\infty}\left(D_{\alpha N}\left(\mathbf{u}^{\prime}\right) \mathfrak{I}_{\alpha N}\left(\mathbf{u}^{\prime}\right) / D_{\alpha N}\left(\mathbf{u}^{\prime}\right)\right) d \alpha\right\} / \sum_{N=-\infty}^{\infty} \int_{-\infty}^{\infty} D_{\alpha N}\left(\mathbf{u}^{\prime}\right) d \alpha \\
& \leq \operatorname{Max}_{\alpha, N}\left\{\operatorname{Max}_{\mathbf{u}^{\prime} \in H} \frac{\mathscr{g}_{\alpha N}\left(\mathbf{u}^{\prime}\right)}{D_{\alpha N}\left(\mathbf{u}^{\prime}\right)}\right\}=\operatorname{Max}_{\alpha, N} \rho^{-1}(\alpha, N) .
\end{aligned}
$$

(This relation is a trivial modification of a lemma of Velte [7].) On the other hand, for the maximizing element $\mathfrak{u}^{\prime}=\tilde{\mathbf{u}}^{\prime}(r, \alpha, N)$ we have, using (12c), that

$$
\operatorname{Max}_{\alpha, N} \rho^{-1}(\alpha, N)=\frac{\mathscr{g}_{\alpha N}\left(\tilde{\mathbf{u}}^{\prime}\right)}{D_{\alpha N}\left(\tilde{\mathbf{u}}^{\prime}\right)}=\frac{I_{\alpha N}(\tilde{\mathbf{u}})}{D_{\alpha N}(\tilde{\mathbf{u}})}=\frac{I(\tilde{\mathbf{v}})}{D(\tilde{\mathbf{v}})} \leq \frac{1}{R} .
$$

The last two inequalities can hold only if

$$
1 / R=\operatorname{Max}_{\alpha, N} \rho^{-1}(\alpha, N) .
$$

Given what has been said about the existence of functions $\mathfrak{u}^{\prime} \in H$ and finite values $(\alpha, N) \neq(0,0)$ which solve the maximum problems, this last result also holds relative to periodic functions of class (12a).

In the analysis carried out below we shall assume, but do not prove, that elements $\mathbf{u}^{\prime}$ which solve the maximum problem are regular solutions of the Euler equations (5). Relative to (12a) or (12b) we have (after eliminating $p$ )

$$
\begin{aligned}
& \rho r w=-L_{N} u+(\alpha r / N) \mathfrak{L}_{N} v+2 i \alpha(w / r), \\
& \text { рru }=-\mathfrak{L}_{N} w+2 D(w / r)-(i / N)\left[D\left(r \aleph_{N} v\right)-\left(2 N^{2} / r^{2}\right) v\right], \\
& D(w r)+i N v+i \alpha r u=0, \\
& u, v, w=0 \text { (a) } r=1 \text {, } \\
& u, v, w \text { are bounded at } r=0 \text {. }
\end{aligned}
$$

These are also Euler equations for the eigenvalues

$$
1 / \rho(\alpha, N)=I_{\alpha N}(\mathfrak{u}) / D_{\alpha N}(\mathfrak{u})=\mathscr{g}_{\alpha N}\left(\mathfrak{u}^{\prime}\right) / D_{\alpha N}\left(\mathfrak{u}^{\prime}\right)
$$

over $\mathbf{u}$ satisfying (18c) and (18d) or $\mathbf{u}^{\prime}$ satisfying only (18d) (and bounded in norm). The Euler equations $(18 \mathrm{a}, \mathrm{b})$ are obtained as the coefficients of $\delta u^{*} \delta w^{*}$ after expressing the dependence of $\delta v^{*}$ on the independent variations through the continuity equation (18c). Direct elimination of the side constraint is a procedure alternate to the introduction of Lagrange multipliers in the calculus of variations. The direct procedure leads to an unsymmetrical form of Euler's equations which are natural to the problem and are not readily suggested from the symmetric form of Euler's equations which comes out of multiplier procedures.

In this way we have reduced the problem of stability to the study of the

2. Eigenvalues $\rho(\alpha, N)$. It is convenient to relax the requirement that $N$ take on only integer values and to consider continuous $N$. Then the stability limit $R$ is contained in that subset of the surface $\rho(\alpha, N)$ belonging to integer values $N$. 
Our first result guarantees that there exists a supremum, independent of $\alpha$ and $N$ for the values $\rho^{-1}$ and establishes that $\rho \rightarrow \infty$ as $\alpha^{2}+N^{2} \rightarrow \infty$.

Lemma 1. Let $\alpha$ and $N$ be nonnegative real numbers and let $\rho(\alpha, N)$ be any eigenvalue of (18). Then

$$
1 / \rho(\alpha, N) \leq 1 /\left(\lambda+N^{2}+\alpha^{2}-2 \alpha\right)
$$

where $\lambda^{1 / 2}=2.405$ is the first zero of $J_{0}\left(\lambda^{1 / 2}\right)$.

In Lemma 3 we show it is sufficient for all $\alpha$ and $N$ to consider only nonnegative values.

Proof. The inequalities

and

$$
\begin{aligned}
0 & \leq r \leq 1 \\
\left|\left\langle r u w^{*}\right\rangle \pm\left\langle r u^{*} w\right\rangle\right| & \leq\left\langle r|u|^{2}\right\rangle+\left\langle r|w|^{2}\right\rangle
\end{aligned}
$$

$$
\left\langle|D \varphi|^{2}\right\rangle \geq \lambda\left\langle\left|\varphi^{2}\right|\right\rangle
$$

for $\varphi$ satisfying (18d, e), are used in the proof. We start with an estimate of $D_{\alpha N}$ from below.

$$
\begin{aligned}
D_{\alpha N}= & \left\langle|D w|^{2}+|D v|^{2}+|D u|^{2}+\alpha^{2}\left(|w|^{2}+|v|^{2}+|u|^{2}\right)+N^{2}|u / r|^{2}\right\rangle \\
& +\left\langle|N w+i v|^{2} / r^{2}+|N v-i w|^{2} / r^{2}\right\rangle \geq\left(\lambda+\alpha^{2}\right)\left\langle|w|^{2}\right\rangle+\left(\lambda+\alpha^{2}+N^{2}\right)\left\langle|u|^{2}\right\rangle \\
& +\left\langle|N w+i v|^{2}+|N v-i w|^{2}\right\rangle \geq\left(\lambda+\alpha^{2}+N^{2}+1\right)\left\langle|w|^{2}\right\rangle \\
& +\left(\lambda+\alpha^{2}+N^{2}\right)\left\langle|u|^{2}\right\rangle-2 i N\left(\left\langle v^{*}, w\right\rangle-\left\langle v, w^{*}\right\rangle\right) .
\end{aligned}
$$

Multiply (18c) by $r w^{*}$, add this to its complex conjugate and integrate to produce the relation

Then

$$
i N\left[\left\langle v w^{*}\right\rangle-\left\langle v^{*} w\right\rangle\right]+i \alpha\left[\left\langle r u w^{*}\right\rangle-\left\langle r u^{*} w\right\rangle\right]=0 .
$$

$$
\begin{aligned}
i N\left(\left\langle v w^{*}\right\rangle-\left\langle v^{*} w\right\rangle\right) & =-i \alpha\left(\left\langle r u w^{*}\right\rangle-\left\langle r u^{*} w\right\rangle\right) \\
& \geq-\alpha\left\langle r|u|^{2}\right\rangle-\alpha\left\langle r|w|^{2}\right\rangle .
\end{aligned}
$$

Eqs. (23c) and (24b) are combined to produce the estimate

$$
\begin{aligned}
D_{\alpha N} & \geq\left(\lambda+\alpha^{2}+N^{2}+1-2 \alpha\right)\left\langle r|w|^{2}\right\rangle+\left(\lambda+\alpha^{2}+N^{2}-2 \alpha\right)\left\langle r|u|^{2}\right\rangle \\
& \geq\left(\lambda+\alpha^{2}+N^{2}-2 \alpha\right)\left\{\left\langle r|w|^{2}\right\rangle+\left\langle r|u|^{2}\right\rangle\right\} .
\end{aligned}
$$

On the other hand $I_{\alpha N} \leq\left\langle r|w|^{2}\right\rangle+\left\langle r|u|^{2}\right\rangle$ and Lemma 1 follows.

Our next result establishes that $\rho \rightarrow \infty$ as $\alpha^{2}+N^{2} \rightarrow 0$.

Lemma 2. Let $\rho(\alpha, N)$ be any eigenvalue of (18). Then

$$
\frac{1}{\rho(\alpha, N)} \leq \frac{2^{3 / 2}}{(\lambda+1)^{1 / 2}}\left(\frac{\alpha^{2}}{\lambda^{2}}+\frac{N^{2}}{2 \lambda(N-1)^{2}}\right)^{1 / 2} .
$$

Proof. Again estimate $D_{\alpha N}$ from below

$$
\begin{aligned}
D_{\alpha N} & \geq\left\langle|D u|^{2}\right\rangle+\left(N^{2}+1\right)\left\langle|w / r|^{2}+|v / r|^{2}\right\rangle-2 i N\left(\left\langle v^{*} / r, w / r\right\rangle-\left\langle w^{*} / r, v / r\right\rangle\right) \\
& \geq\left\langle|D u|^{2}\right\rangle+(N-1)^{2}\left\langle|w / r|^{2}+|v / r|^{2}\right\rangle \\
& \geq \lambda\left\langle|u|^{2}\right\rangle+(N-1)^{2}\left\langle|v / r|^{2}\right\rangle .
\end{aligned}
$$


For $I_{\alpha N}$ we use Schwarz's inequality

$$
I_{\alpha N} \leq 2\left(\left\langle r|u|^{2}\right\rangle\left\langle r|w|^{2}\right\rangle\right)^{1 / 2} \leq 2\left(\left\langle|u|^{2}\right\rangle\left\langle|w|^{2}\right\rangle\right)^{1 / 2} .
$$

It is easily established from Eq. (18c) that

$$
\left\langle|D w|^{2}\right\rangle+\left\langle|w / r|^{2}\right\rangle=\left\langle|N v / r+\alpha u|^{2}\right\rangle
$$

which leads to the estimate

$$
(\lambda+1)\left\langle|w|^{2}\right\rangle \leq 2 \alpha^{2}\left\langle|u|^{2}\right\rangle+2 N^{2}\left\langle|v / r|^{2}\right\rangle .
$$

The estimates (27) and (28) are combined into

$$
I_{\alpha, v} \leq \frac{2^{3 / 2}}{(\lambda+1)^{1 / 2}}\left(\alpha^{2}\left\langle|u|^{2}\right\rangle^{2}+N^{2}\left\langle|u|^{2}\right\rangle\left\langle\left|\frac{v}{r}\right|^{2}\right\rangle\right)^{1 / 2} .
$$

Now combine (26) and (29) to produce

$$
\begin{aligned}
\frac{I_{\alpha N}}{D_{\alpha N}} & \leq \frac{2^{3 / 2}}{(\lambda+1)^{1 / 2}}\left(\frac{\alpha^{2}\left\langle|u|^{2}\right\rangle^{2}+N^{2}\left\langle|u|^{2}\right\rangle\left\langle|v / r|^{2}\right\rangle}{\left(\lambda\left\langle|u|^{2}\right\rangle+(N-1)^{2}\left\langle|v / r|^{2}\right\rangle\right)^{2}}\right)^{1 / 2} \\
& \leq \frac{2^{3 / 2}}{(\lambda+1)^{1 / 2}}\left(\frac{\alpha^{2}}{\lambda^{2}}+\frac{N^{2}}{2 \lambda(N-1)^{2}}\right)^{1 / 2}
\end{aligned}
$$

proving Lemma 2.

Now introduce polar coordinates in wave number space

$$
\alpha=d \sin \varphi, \quad N=\cdot d \cos \varphi, \quad d=\left(\alpha^{2}+N^{2}\right)^{1 / 2} .
$$

We have established that along any $\operatorname{ray} \varphi=$ const there exists a finite nonzero value of $d$ on which $\rho^{-1}$ is a maximum. Let $\rho^{-1}(\alpha, N)=\mu(d, \varphi)$. Let $d=d(\varphi)$ be that value of $d$ for which the relation

$$
\tilde{\rho}^{-1}=\tilde{\mu}(\varphi)=\mu(d(\varphi), \varphi)=\operatorname{Max}_{d>0} \mu(d, \varphi)
$$

holds.

We next establish a result which allows us to restrict the search for the value $R$ to the first quadrant $\varphi \in[0, \pi / 2]$ and also shows that $\varphi=0$ and $\varphi=\pi / 2$ are locally extremes of the curve $\tilde{\mu}(\varphi)$.

Lemma 3. $\rho(\alpha, N)$ is an even function of $\alpha$ and $N$.

Proof. From (19) it is obvious that $\rho$ is real-valued. If $u, v$, and $w$ are eigenfunctions belonging to $\rho$ so too are $u^{*}, v^{*}$ and $w^{*}$ eigenfunctions of $\rho$.

First we prove symmetry with respect to $N$. We note that Eqs. (18) unaltered under the transformation

$$
\begin{array}{ll}
w(r, \alpha, N) & -w(r, \alpha,-N) \\
v(r, \alpha, N) \rightarrow & v(r, \alpha,-N) \\
u(r, \alpha, N) & -u(r, \alpha,-N)
\end{array}
$$

These new functions are then eigenfunctions of the same value $\rho$. That this is the value $\rho(\alpha,-N)$ follows upon insertion of the transformation into (19). With respect to $\alpha$ symmetry we have invariance for (18) under 


$$
\begin{array}{lr}
w(r, \alpha, N) & -w^{*}(r,-\alpha, N) \\
v(r, \alpha, N) & \rightarrow \quad v^{*}(r,-\alpha, N) \\
u(r, \alpha, N) & -u^{*}(r,-\alpha, N) .
\end{array}
$$

That $\rho(\alpha, N)=\rho(-\alpha, N)$ follows from this transformation and (19). This proves Lemma 3.

It is clear that all of the values of $\rho^{-1}(\alpha, N)=\mu(d, \varphi)$ are to be found in the first quadrant $\varphi \in[0, \pi / 2]$. By Lemmas 1 and 2 we know that a maximum value $\tilde{\rho}^{-1}=$ $\tilde{\mu}(\varphi)=\mu(d(\varphi), \varphi)$ for $0<d(\varphi)<\infty$, exists. The value $d(\varphi)$ is then a root of

$$
(\partial \mu / \partial d)_{d=d|\varphi|}=0 \text {. }
$$

These maximum values $\tilde{\mu}$ form a line of mountain tops depending on the angle $\varphi$. On this line, by the chain rule and (34)

$$
\frac{d \tilde{\mu}}{d \varphi}=\frac{\partial \mu}{\partial d} \frac{d(d)}{d \varphi}+\frac{\partial \mu}{\partial \varphi}=\frac{\partial \mu}{\partial \varphi}=-\frac{1}{\rho^{2}}\left[\frac{\partial \rho}{\partial \alpha} N-\frac{\partial \rho}{\partial N} \alpha\right] .
$$

Then, from the symmetries of $\rho$,

$$
d \tilde{\mu} / d \varphi \quad(\varphi=\pi / 2)=0, \quad d \tilde{\mu} / d \varphi \quad(\varphi=0)=0 .
$$

It follows that $\varphi=\pi / 2$ and $\varphi=0$ are extremes of $\tilde{\mu}(\varphi)=\tilde{\rho}^{-1}$. Orr has found that $\tilde{\mu}(\pi / 2)=$ $(180)^{-1}$. We find that $\mu(d(0), 0)=\tilde{\mu}(0)=(82.54)^{-1}$ where $d(0)=1.13$. Of course, the stability limit necessarily occurs among integers $N$. For $N=1$ we have $\mu(1,0)=(82.88)^{-1}$. It turns out that this value is a local minimum and that the true maximizing value is associated with a third stationary point $\alpha=1.07, N=1, \varphi \approx \pi / 4$.

In the work which follows it is convenient to introduce the variable $f=w r$. It is also convenient to work with the pair of differential equations

$$
\begin{array}{r}
\rho N^{2} f=i \alpha r D L f-\left(N^{2}+\alpha^{2} r^{2}\right) L u-2 \alpha^{2} r D u, \\
\rho\left[\left(N^{2}+\alpha^{2} r^{2}\right) u+i \alpha r D f\right]=L^{2} f+4 i \alpha L u,
\end{array}
$$

and boundary conditions

$$
f=D f=u=0 \quad \text { at } r=1, \quad f, u \text { bounded at } r=0,
$$

which are equivalent to the set (18). To derive the set (37) from (18), substitute $f$ in (18), then eliminate $v$ with $(18 \mathrm{c})$ and rearrange to produce

$$
\begin{gathered}
\rho N^{2} f=i \alpha r D L f-\left(N^{2}+\alpha^{2} r^{2}\right) L u-2 \alpha^{2} r D u, \\
\rho N^{2} u-\left(\alpha^{2} L f+2 i \alpha^{3} u+i \alpha r D L u\right)=L^{2} f+4 i \alpha L u .
\end{gathered}
$$

The first of these equations is identical to (37a). To obtain (37b) differentiate (38a) once, multiply the result by $i \alpha r / N^{2}$ and add to (38b) multiplied by $\alpha^{2} r^{2} / N^{2}$. This leads to

$$
\rho\left(i \alpha r D f+\alpha^{2} r^{2} u\right)=-\left(\alpha^{2} L f+2 i \alpha^{3} u+i \alpha r D L u\right) .
$$

Eq. (37b) then follows from substituting (39) into (38b).

3. Eigenvalues $\rho(\alpha, 0)$-Orr's problem. Orr solves (37a), (37b) and (37c) when $N=0$. Then, whether or not $v$ is set to zero we have from (18c)

$$
D f=-i \alpha r u \text {. }
$$


Eq. (40) reduces (37a) to an identity and leads through (37b) to

$$
L^{2} f-4 L(D f / r)=2 i \alpha \rho r D f,
$$

which can be re-expressed

$$
D^{2} f=2 i \alpha \rho r D f,
$$

where $D=D^{2}-(1 / r) D-\alpha^{2}$. The values

$$
180 \cong \operatorname{Min}_{\alpha} \rho(\alpha, 0), \quad \alpha \cong 3.7,
$$

have been obtained from (37c) and (41a) by Orr. These values compare with the values

$$
180.6=\underset{\alpha}{\operatorname{Min} \rho(\alpha, 0),} \quad \alpha=3.6,
$$

which are obtained by numerical integration. There is a difficulty, associated with the singular coefficients of (41a), in using the efficient Runge-Kutta-Gill forward integration procedure. This difficulty we overcome by starting the integration from values of the solution known near the origin through Frobenius expansions. An account of this method is included as an appendix to this paper. Orr has used the Frobenius expansions to represent the solution everywhere in $r \in[0,1]$ and the remarkably accurate values of (42) are obtained from estimates of these solutions and hand calculations.

The value $\rho=180.6$ cannot be the minimum eigenvalue for (37a), (37b) and (37c) because

$$
\rho(0,1)=82.88 \text {. }
$$

To demonstrate this we now consider the

4. Eigenvalues $\rho(0, N)$. When $\alpha=0$ we have the relatively simple real system

$$
\begin{gathered}
L^{2} f-\rho N^{2} u=0, \\
L u+\rho f=0, \\
L=\frac{1}{r} D(r D)-\frac{N^{2}}{r^{2}}, \\
f=D f=u=0 \quad \text { at } \quad r=1, f, u \text { bounded at } r=0 .
\end{gathered}
$$

It is convenient at this point to reformulate (45), (46) and (47) as an integral equation. For this purpose we use the Green's functions for

$$
\begin{gathered}
L G=0, \quad r \in(0,1), r \neq r_{0}, \\
G\left(r, r_{0} ; N\right)=G\left(r_{0}, r ; N\right), \\
D G\left(r_{0}+, r_{0} ; N\right)-D G\left(r_{0}, r_{0}-; N\right)=-1 / r \\
G\left(1, r_{0} ; N\right)=0, \\
G\left(0, r_{0} ; N\right) \text { bounded, }
\end{gathered}
$$

and for 


$$
\begin{gathered}
L^{2} H=0, \quad r \in(0,1), r \neq r_{0}, \\
H\left(r, r_{0} ; N\right)=H\left(r_{0}, r ; N\right), \\
D H\left(r_{0}+, r_{0} ; N\right)=D H\left(r_{0}, r_{0}-; N\right), \\
D^{2} H\left(r_{0}+, r_{0} ; N\right)=D^{2} H\left(r_{0}, r_{0}-; N\right), \\
D^{3} H\left(r_{0}+, r_{0} ; N\right)-D^{3} H\left(r_{0}, r_{0}-; N\right)=-1 / r, \\
H\left(1, r_{0} ; N\right)=0, \\
D H\left(1, r_{0} ; N\right)=0, \\
H\left(0, r_{0} ; N\right) \text { bounded, }
\end{gathered}
$$

which are given by

$$
G=-(1 / 2 N)\left\{\left(r r_{0}\right)^{N}-\left(r_{0} / r\right)^{N}\right\}, \quad r>r_{0},
$$

and

$$
\begin{aligned}
H=\left(1 / 8 N\left(N^{2}-1\right)\right)\{(N & -1)\left(r r_{0}\right)^{N} r^{2}\left[N r_{0}^{2}-(N+1)\right] \\
& +(N+1)\left(r_{0} r\right)^{N}\left[(1-N) r_{0}^{2}+N\right]-(N+1)\left(r_{0} / r\right)^{N} r^{2} \\
& \left.+(N-1)\left(r_{0} / r\right)^{N} r_{0}^{2}\right\}, \quad r>r_{0} .
\end{aligned}
$$

It can be verified by inspection ${ }^{3}$ of (50) and (51) that

$$
\begin{array}{ll}
G\left(r, r_{0} ; N\right)>0, & r, r_{0} \in(0,1), \\
H\left(r, r_{0} ; N\right)<0, & r, r_{0} \in(0,1) .
\end{array}
$$

To obtain an integral equation for $f$ we first re-express (45), (46) and (47) with (50) and (51). Thus

$$
u=\rho \int_{0}^{1} G\left(r, r_{0} ; N\right) f\left(r_{0}\right) d r_{0}
$$

and

$$
f=-\rho N^{2} \int_{0}^{1} H\left(r, r_{0} ; N\right) u\left(r_{0}\right) d r_{0}
$$

This leads to

$$
f(r ; N)=\rho^{2} \int_{0}^{1} K\left(r, r_{0} ; N\right) f\left(r_{0}, N\right) d r_{0},
$$

where

$$
K\left(r, r_{0} ; N\right)=-N^{2} \int_{0}^{1} H(r, \tau ; N) G\left(\tau, r_{0} ; N\right) d \tau .
$$

From (52), (53) and (57) it follows that

$$
K\left(r, r_{0} ; N\right)>0, r, r_{0} \in(0,1) .
$$

${ }^{3}$ This result is true also for the more general operator

$$
L=a(r) D^{2}+b(r) D+c(r), \text { with } a>0, c<0 .
$$

(see Kirchgässner [8]). 
We have now established elements preliminary to a proof of

Lemma 4. There exists for each fixed $N$ a positive least eigenvalue $\rho^{2}$ of (56). This eigenvalue is simple. The associated eigenfunction $f$ is positive in $(0,1)$. The eigenfunction $u$ belonging to $\rho=+\left(\rho^{2}\right)^{1 / 2}$ is positive. There are no other positive solutions of (45), (46) and (47) belonging to $\rho>0$.

Proof. (56) is an integral equation with a positive kernel. A theorem of Jentzsch [9] applies to such integral equations and guarantees the simplicity of $\rho^{2}$ and the positivity of $f$. The positivity of $u$ follows by inspection of (54) and (52). There is not a second set of positive eigenfunction $f_{2}, u_{2}$ belonging to another eigenvalue $\rho_{2}>0$. Assume the contrary. Then, from the equations

$$
L^{3} f_{1}+\rho_{1}^{2} N^{2} f_{1}=0, \quad L^{3} f_{2}+\rho_{2}^{2} N^{2} f_{2}=0,
$$

we obtain in the usual way

$$
\rho_{2}^{2}\left\langle L^{2} f_{1}, f_{2}\right\rangle=\rho_{1}^{2}\left\langle L^{2} f_{2}, f_{1}\right\rangle=\rho_{1}^{2}\left\langle L^{2} f_{1}, f_{2}\right\rangle .
$$

The last equality following the property selfadjointness for functions which vanish along with their first derivative at $r=1$. Since by $(45) L^{2} f_{1}$ is positive in $(0,1)$ and since $f_{2}$ is positive in $(0,1)$ by hypothesis we are led to the contradiction $\rho_{2}=\rho_{1}$. This proves the lemma.

We next obtain these unique positive solutions and calculate the smallest values of $\rho(0, N)>0$. It is easiest to start from the system

$$
\begin{gathered}
L^{3} f+\rho^{2} N^{2} f=0, \\
f=D f=L^{2} f=0 \text { at } \quad r=1, \\
f \text { bounded at } r=0 .
\end{gathered}
$$

Then we note that Bessel's function $\varphi=J_{N}\left((\lambda)^{1 / 2} r\right)$ satisfies the equations

$$
\begin{aligned}
L \varphi & =-\lambda \varphi, \\
L^{2} \varphi & =\lambda^{2} \varphi, \\
L^{3} \varphi & =-\lambda^{3} \varphi,
\end{aligned}
$$

and is bounded at the origin. The linear combination

$$
f=A_{1} J_{N}\left(\lambda_{1}^{1 / 2} r\right)+A_{2} J_{N}\left(\lambda_{2}^{1 / 2} r\right)+A_{2}^{*} J_{N}\left(\lambda_{3}^{1 / 2} r\right),
$$

is the general solution of (59) provided that

$$
\lambda_{1}=\left(\rho^{2} N^{2}\right)^{1 / 3}, \quad \lambda_{2}=\lambda_{1} e^{-i(2 / 3) \pi}, \quad \lambda_{3}=\lambda_{1} e^{i(2 / 3) \pi},
$$

and that

$$
F(\rho, N)=\left|\begin{array}{ccc}
J_{N}\left(\lambda_{1}^{1 / 2}\right) & J_{N}\left(\lambda_{2}^{1 / 2}\right) & J_{N}\left(\lambda_{3}^{1 / 2}\right) \\
\lambda_{1}^{1 / 2} D J_{N}\left(\lambda_{1}^{1 / 2}\right) & \lambda_{2}^{1 / 2} D J_{N}\left(\lambda_{2}^{1 / 2}\right) & \lambda_{3}^{1 / 2} D J_{N}\left(\lambda_{3}^{1 / 2}\right) \\
\lambda_{1}^{2} J_{N}\left(\lambda_{1}^{1 / 2}\right) & \lambda_{2}^{2} J_{N}\left(\lambda_{2}^{1 / 2}\right) & \lambda_{3}^{2} J_{N}\left(\lambda_{3}^{1 / 2}\right)
\end{array}\right|=0,
$$

where $F(\rho, N)$ is the determinant of coefficients of $A_{1}, A_{2}$ and $A_{2}^{*}$ in the linear equations 
formed from (60) and $f(1)=D f(1)=L^{2} f(1)=0$. We can rewrite (62) as

$$
\begin{aligned}
& \mathcal{S}(\rho, N)=\frac{F(\rho, N)}{i \sqrt{3} a^{5}}=J_{N}(a) J_{N}\left(a e^{-i \pi / 3}\right) J_{N}\left(a e^{i \pi / 3}\right) \\
& \cdot\left\{\frac{D J_{N}(a)}{J_{N}(a)}+\frac{D J_{N}\left(a e^{-i \pi / 3}\right)}{e^{-\pi i / 3} J_{N}\left(a e^{-i \pi / 3}\right)}+\frac{D J_{N}\left(a e^{i \pi / 3}\right)}{e^{i \pi / 3} J_{N}\left(a e^{i \pi 3}\right)}\right\}=0,
\end{aligned}
$$

where $a=\lambda_{1}^{1 / 2}$.

Our problem is now reduced to an investigation of the roots $\rho$ of (63). We proceed as follows: $\mathcal{G}(\rho, N)$ is a real function of complex argument. For each fixed $N=1,2,3 \cdots$ we seek the smallest positive root of $\mathcal{G}(\rho, N)=0$. We then verify that the corresponding eigenfunctions $f$ and $u$ are positive. Lemma 4 then guarantees that this least root of $\mathcal{G}(\rho, N)=0$ is also the smallest of the eigenvalues $\rho(0, N)$ of (45), (46) and (47). The results which follow were calculated from the Bessel's functions and checked against direct forward integration of (45), (46) and (47) by the Runge-Kutta-Gill method

\section{TABLE I}

Principal eigenvalues of $\rho(0, N)$ of (45), (46) and (47). These eigenvalues are found as the smallest roots, for preassigned $N$, of Eq. (63). Only integer values of $N$ correspond to accepatble (single-valued) solutions.

\begin{tabular}{l|r}
\multicolumn{1}{c|}{$N$} & $\rho(0, N)$ \\
\hline 0.5 & 98.55 \\
1 & 82.88 \\
1.13 & 82.54 \\
2 & 91.04 \\
3 & 110.19 \\
4 & 134.03 \\
5 & 161.27 \\
6 & 191.44
\end{tabular}

and are in perfect agreement (see Appendix). The graph of $f$ and $u$ for $N=1$ is given in Fig. 1. For $N=1$, the coefficients for (60) are

$$
A_{1}=1, \quad A_{2}=0.00301-i 0.02455 .
$$

6. Perturbation of the eigenvalues $\rho(0, N)$. At an early stage of this investigation we conjectured that the value 82.88 was the smallest of the values $\rho(\alpha, N)$ for integers $N$. The basis for such a conjecture is this. The value 82.88 is the smallest value for integer $N$ of the eigenvalues $\rho(0, N)$. This value is a local extremum. For large $\alpha$ and any fixed $N$ it is known from Lemma 1 that $\rho$ is large. In fact, it is to be expected that for any fixed $N$ the eigenvalues $\rho(\alpha, N) \rightarrow \rho(\alpha, 0)$ as $\alpha \rightarrow \infty$ (Orr's solution is the limiting $\alpha \rightarrow \infty$ solution for any $N$ ). For fixed $N$ the local extremum at $\alpha=0$ could be an absolute minimum but could not be an absolute maximum. An even stronger argument, of the same kind, has been given in Sec. 2. From that argument we know that on each $\operatorname{ray} \varphi=$ $\tan ^{-1} N / \alpha$ there exists a minimum value of $\rho=\rho(d(\varphi), \varphi)$ for $0<d=\left(\alpha^{2}+N^{2}\right)^{1 / 2}<\infty$. The intersection of the line $d(\varphi)$ of minimum values with the $\alpha=0$ and $N=0$ axes locates local extreme points of $\rho$ on the line of minimum values $d(\varphi)$ and, on this line, $\rho(\alpha, 0)>\rho(0, N)$. If the variation were monotonic $\rho(0, N)$ would be globally minimum. 


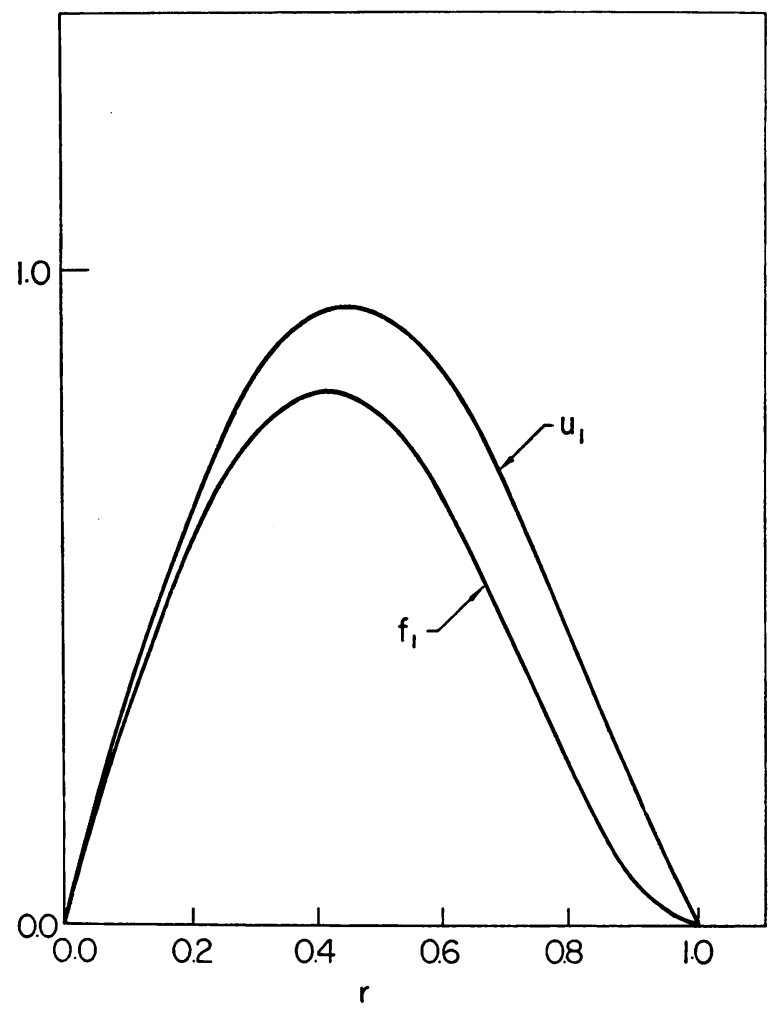

FIG. 1. Principal eigenfunction $f(r, 1), u(r, 1)$ of Eqs. (45), (46), and (47).

Moreover, our extended calculations for the annulus (Sec. 8) show that among integers $N$ the minimizing solutions are purely azimuthal (transverse to the main stream) for radius ratios as small as $10^{-4}$. For the plane Couette flow this result has been obtained numerically (Busse [10]). It is consistent with the results of Serrin [4] for Couette flow between cylinders when the gap is narrow, and when the gap is large [11] and is can be proved for plane Couette flow [5].

But the conjecture is false, and $\rho(0, N)$ cannot be the smallest of the values $\rho(\alpha, N)$ for all (continuous) $N \neq 0$. It is known from Orr's calculations and from ours that over $\alpha$ the Min $\rho(\alpha, 0)=\rho(3.6,0)=180.6$, and $\rho(\alpha, 0) \rightarrow \infty$ as $\alpha \rightarrow 0$. Now consider $\rho(\alpha, N)$ for very small continuous $N=\epsilon$. It is known that eigenvalues exist for $\epsilon \neq 0, \alpha=0$ so that, by Lemma $2, \rho(0, \epsilon)$ can be made arbitrarily large by decreasing $\epsilon$. By Orr's calculation $\rho(3.6,0)=180.6$ and, by continuity $\rho(3.6, \epsilon) \cong 180.6$. For small $N=\epsilon$, then, $\rho(\alpha, \epsilon)$ decreases as $\alpha$ is increased from 0 to 3.6. Since $\partial \rho(0, \epsilon) / \partial \alpha=0$, it is to be expected that $\partial^{2} \rho(0, \epsilon) / \partial \alpha^{2}<0$. This expectation is correct, it turns out, but the true situation is a delicate one; $\rho(0, N)$ changes from a local maximum (on the curve $N=$ const) to a local minimum as $N$ is increased from $N=1$ to $N=2$.

In the lemma which follows the results of the $\alpha$ perturbation of the exact solution of Sec. 4 are summarized. We use the notation $g^{(\nu)} \equiv \partial^{\nu} g /\left.\partial \alpha^{\nu}\right|_{\alpha=0}$ for any $C^{\nu}$ function of $d$ and consider solutions $f(r, \alpha, N), u(r, \alpha, N)$ and $\rho(\alpha, N)$ of Eqs. (37).

Lemma 5. Eqs. (37) are uniquely solvable when $\alpha=0$ (Lemma 4). The unique solu- 
tion is (see Sec. 4)

$$
f(r, 0, N)=\sum_{i=1}^{3} A_{i} J_{N}\left(\lambda_{i}^{1 / 2} r\right), \quad u(r, 0, N)=\rho \sum_{i=1}^{3} A_{i} \lambda_{i}^{-1} J_{N}\left(\lambda_{i}^{1 / 2} r\right)
$$

where the $A_{i}$ are chosen so as to satisfy (37c) and

$$
\left\langle f^{2}\right\rangle+\left\langle u^{2}\right\rangle=1 \text {. }
$$

There is a unique $\alpha$ analytic branch of (37) passing through $\alpha=0$. On this branch

$f^{(1)}(r, 0, N)=\frac{i \rho}{6} \sum_{i=1}^{3} A_{i} \lambda_{i}^{-1}\left(1-r^{2}\right) J_{N}\left(\lambda_{i}^{1 / 2} r\right)$,

$u^{(1)}(r, 0, N)=\frac{i}{6 N^{2}} \sum_{i=1}^{3} A_{i}\left\{\lambda_{i}\left(1-r^{2}\right) J_{N}\left(\lambda_{i}^{1 / 2} r\right)-8 J_{N}\left(\lambda_{i}^{1 / 2} r\right)+2 r D J_{N}\left(\lambda_{i}^{1 / 2} r\right)\right\}$,

$\rho^{(1)}(0, N)=0$,

and

$\rho^{(2)}(0, N)=\left\{\left(N^{2}+2\right)\left\langle u^{2}\right\rangle+N^{-2}\left\langle r^{2}(L f)^{2}\right\rangle+\left(4 N^{-2}-1\right)\langle f, L f\rangle-G^{2}\right\} / N^{2}\langle u f\rangle$

where

$$
-G^{2}=\left\langle\left(L f^{(1)}\right)^{2}\right\rangle-N^{2}\left\langle u^{(1)}, L u^{(1)}\right\rangle-2 \rho N^{2}\left\langle u^{(1)} f^{(1)}\right\rangle \leq 0 .
$$

The equations of the lemma permit the calculation of the values $\rho^{(2)}(0, N)$ given in Table II. Eq. (65a) already suggests that $\rho^{(2)}>0$ when $N \geq 2$, (for if $N \geq 2$, then

\section{TABLE II}

Second derivatives with respect to $\alpha$ of the eigenvalues $\rho(\alpha, N)$ for fixed $N$ at $\alpha=0$. These derivatives are exact values which are obtained from equations of the lemma. At $\alpha=0, \rho^{(1)}(0, N)=0$ for all $N$. The eigenvalues $\rho(0, N)$ are locally maximum when $N>1.21$ and locally minimum when $N<1.20$ (cf. Fig. 2).

$\begin{array}{lr}\quad & \rho^{(2)}(0, N) \\ 0.5 & -167.687 \\ 1 & -\quad 4.429 \\ 1.13 & -1.575 \\ 1.2 & -\quad 0.503 \\ 1.4 & 1.541 \\ 2 & 3.782 \\ 3 & 4.383 \\ 4 & 4.346 \\ 5 & 4.222 \\ 6 & 4.096\end{array}$

$\left.\left(4 N^{-2}-1\right)\langle f, L f\rangle=-\left(4 N^{-2}-1\right)\left\{\left\langle\left(D f^{2}\right)\right\rangle+\left\langle(f / r)^{2}\right\rangle\right\} \geq 0\right)$.

The existence of a unique $\alpha$ analytic perturbation for (37) at $\alpha=0$ is a consequence of the simplicity of the principal eigenvalue (Lemma 4) and Rellich's Theorem (see Kato [12], Chap. 7, for a complete discussion and the relevant references). We calculate the first few $\alpha$ derivatives.

Write Eqs. (37a) and (37b) in matrix notation

$$
\mathbf{A} \cdot \mathbf{q}=\alpha \mathbf{B} \cdot \mathbf{q}
$$


where

$$
\mathbf{q}=\left[\begin{array}{l}
f \\
u
\end{array}\right], \quad \mathbf{A}=\left[\begin{array}{cc}
L^{2} & -\rho N^{2} \\
-\rho N^{2} & -N^{2} L
\end{array}\right]
$$

and

$$
\mathbf{B}=\left[\begin{array}{cc}
i \rho r D & \left(\rho \alpha r^{2}-4 i L\right) \\
-i r D L & \alpha\left(r^{2} L+2 r D\right)
\end{array}\right] .
$$

We make use of the identity

$$
L(r D f) \equiv 2 L f+r D L f
$$

and the relations $L^{(1)}=0\left(L^{(2)}=-2\right)$, and $L U=-\rho f$ which hold when $\alpha=0$ to find after an integration by parts that

$$
\langle\mathbf{q}, \mathbf{B} \cdot \mathbf{q}\rangle=i\{\rho\langle f r D f\rangle-\langle u r D(L f)\rangle-4\langle f L U\rangle\}=0,
$$

when $\alpha=0$.

By direct differentiation of (66) we have for $\alpha=0$

$$
\begin{aligned}
A \cdot q & =0, \\
A \cdot q^{(1)} & =-A^{(1)} \cdot q+B \cdot q,
\end{aligned}
$$

and

$$
A \cdot q^{(2)}=-A^{(2)} \cdot q+2 B^{(1)} \cdot q+2 B \cdot q^{(1)}-2 A^{(1)} \cdot q^{(1)},
$$

where $q, q^{(1)}$ and $q^{(2)}$ all satisfy the same boundary conditions. The $\alpha$ symmetry of $\rho$ implies that $\mathbf{A}^{(1)}=0$. This result is also implied by the orthogonality condition which must be imposed on the right of $(68 b), 0=\left\langle q^{(1)}, A \cdot q\right\rangle=\left\langle q, A \cdot q^{(1)}\right\rangle=-\left\langle q, A^{(1)} \cdot q\right\rangle$, Unfolding of the last scalar product gives $\rho^{(1)}=0$.

Similarly, for the right side of (68c) we have

$$
\begin{aligned}
0 & =\left\langle\mathbf{q}^{(2)}, \mathbf{A} \cdot \mathbf{q}\right\rangle=\left\langle\mathbf{q}, \mathbf{A} \cdot \mathbf{q}^{(2)}\right\rangle \\
& =-\left\langle\mathbf{q}, \mathbf{A}^{(2)} \cdot \mathbf{q}\right\rangle+2\left\langle\mathbf{q}, B^{(1)} \cdot \mathbf{q}\right\rangle+2\left\langle\mathbf{q}, \mathbf{B} \cdot \mathbf{q}^{(1)}\right\rangle .
\end{aligned}
$$

The relations

$$
\left\langle\mathbf{q}, \mathbf{B}^{(1)} \cdot \mathbf{q}\right\rangle=-2\left\langle u^{2}\right\rangle
$$

and

$$
\left\langle\mathbf{q}, \mathbf{A}^{(2)} \cdot \mathbf{q}\right\rangle=-4\langle f, L f\rangle-2 \rho^{(2)} N^{2}\langle u f\rangle+2 N^{2}\left\langle u^{2}\right\rangle,
$$

follow from straightforward calculations. Eqs. (69), (70), and (71) are used to deduce (65), but we must first transform the last term of (69). Consider

$\left\langle\mathbf{q}, \mathbf{B} \cdot \mathbf{q}^{(1)}\right\rangle+\left\langle\mathbf{q}^{(1)}, \mathbf{B} \cdot \mathbf{q}\right\rangle$

$$
=i\left\{\rho\left\langle f r D f^{(1)}\right\rangle+\rho\left\langle f^{(1)} r D f\right\rangle-\left\langle L u, r D f^{(1)}\right\rangle-\left\langle L u^{(1)}, r D f\right\rangle-2\left\langle f L u^{(1)}\right\rangle-2\left\langle f^{(1)} L u\right\rangle\right\} .
$$

In the above eliminate $L u(=-\rho f)$ and $L u^{(1)}\left(=-\rho f^{(1)}+i N^{-2} r D(L f)\right)$ and integrate 
by parts to continue the last equation as

$$
\begin{aligned}
& =N^{-2}\{\langle r D L f, r D f\rangle+2\langle f, r D L f\rangle\}, \\
& =-N^{-2}\left\{\left\langle r^{2}(L f)^{2}\right\rangle+\left(4+N^{2}\right)\langle f L f\rangle\right\} .
\end{aligned}
$$

Finally, using $(68 \mathrm{~b})$, one finds that

$$
\left\langle\mathbf{q}, \mathbf{B} \cdot \mathbf{q}^{(1)}\right\rangle=-\left\langle\mathbf{q}^{(1)}, \mathbf{A} \cdot \mathbf{q}^{(1)}\right\rangle-N^{-2}\left\{\left\langle r^{2}(L f)^{2}\right\rangle+\left(4+N^{2}\right)\langle f, L f\rangle\right\} \text {. }
$$

Here, $q^{(1)}=i \mathbf{q}^{\prime}$ where $q^{\prime}$ is real and $\left\langle\mathbf{q}^{\prime}, \mathbf{A} \cdot \mathbf{q}^{\prime}\right\rangle=G^{2}$. Eqs. (69-72) are now combined to form (65a).

To prove the variational inequality $(65 \mathrm{~b})$ note that

$$
\frac{2 N^{2}\left\langle u^{\prime} f^{\prime}\right\rangle}{\left\langle\left(L f^{\prime}\right)^{2}\right\rangle-N^{2}\left\langle u^{\prime} L u^{\prime}\right\rangle} \leq \frac{1}{\rho}
$$

for any admissible $f^{\prime}$ and $u^{\prime}$ with equality holding for $u^{\prime}=u, f^{\prime}=f$. In matrix notation $\left\langle\mathbf{q}^{\prime}, \mathbf{A} \cdot \mathbf{q}^{\prime}\right\rangle \geq 0$ for any admissible $\mathbf{q}^{\prime}$. The vector $q^{(1)} / i$ is admissible, proving (65b).

It may be readily verified that the functions given by $(64 a, b)$ reduce Eq. (68b) (with $\mathbf{A}^{(1)} \equiv 0$ ) to an identity. Moreover, this solution satisfies the boundary conditions at $r=1$ and is bounded at $r=0$. The solution is unique on the subspace orthogonal to the solution of the homogeneous problem. This subspace is already implied for solutions of (37) of norm $\langle\mathbf{q}(\alpha) \cdot \overline{\mathbf{q}}(\alpha)\rangle=1$. The lemma is proved.

TheOREM. $\quad R<82.88$.

This is obviously true since $\rho(0,1)=82.88, \rho^{(1)}(0,1)=0$ and $\rho^{(2)}(0,1)<0$.

The value $R=\rho(1.07,1)=81.49$ is a result of the Runge-Kutta integration of Eq. (37). The integration is started with values of independent solutions $f_{1}=r^{N}, f_{2}=r^{N+2}$, $u_{1}=r^{N}$ given by a Frobenius analysis at the origin. The graphs of $\rho(\alpha, N)$, for several $N$ are given in Fig. 2.

7. Axial flow in an annulus. The basic velocity profile governing the rectilinear motion of a viscous fluid down an annulus with inner radius $r_{1}$ and outer radius $r_{0}$ is given by

$$
\mathrm{V}=(W, V, U)=\left(0,0, U_{M}\left[\frac{1-r^{2}(1-\eta)^{2}+r_{c}^{2} \ln r^{2}(1-\eta)^{2}}{1-r_{c}^{2}+r_{c}^{2} \ln r_{c}^{2}}\right]\right)
$$

where

$$
r=r^{*} / d, \quad d=r_{0}-r_{1}, \quad \eta=r_{1} / r_{0}, \quad r_{c}^{2}=\left(\eta^{2}-1\right) / \ln \eta^{2} .
$$

The deformation matrix for this motion is given by

$$
\mathbf{E}=-g(r ; \eta) r\left(\begin{array}{lll}
0 & 0 & 1 \\
0 & 0 & 0 \\
1 & 0 & 0
\end{array}\right)
$$

where

$$
g(r ; \eta)=\frac{\left[(1-\eta)^{2}-r_{c}^{2} / r^{2}\right]}{1-r_{c}^{2}+r_{c}^{2} \ln r_{c}^{2}}
$$




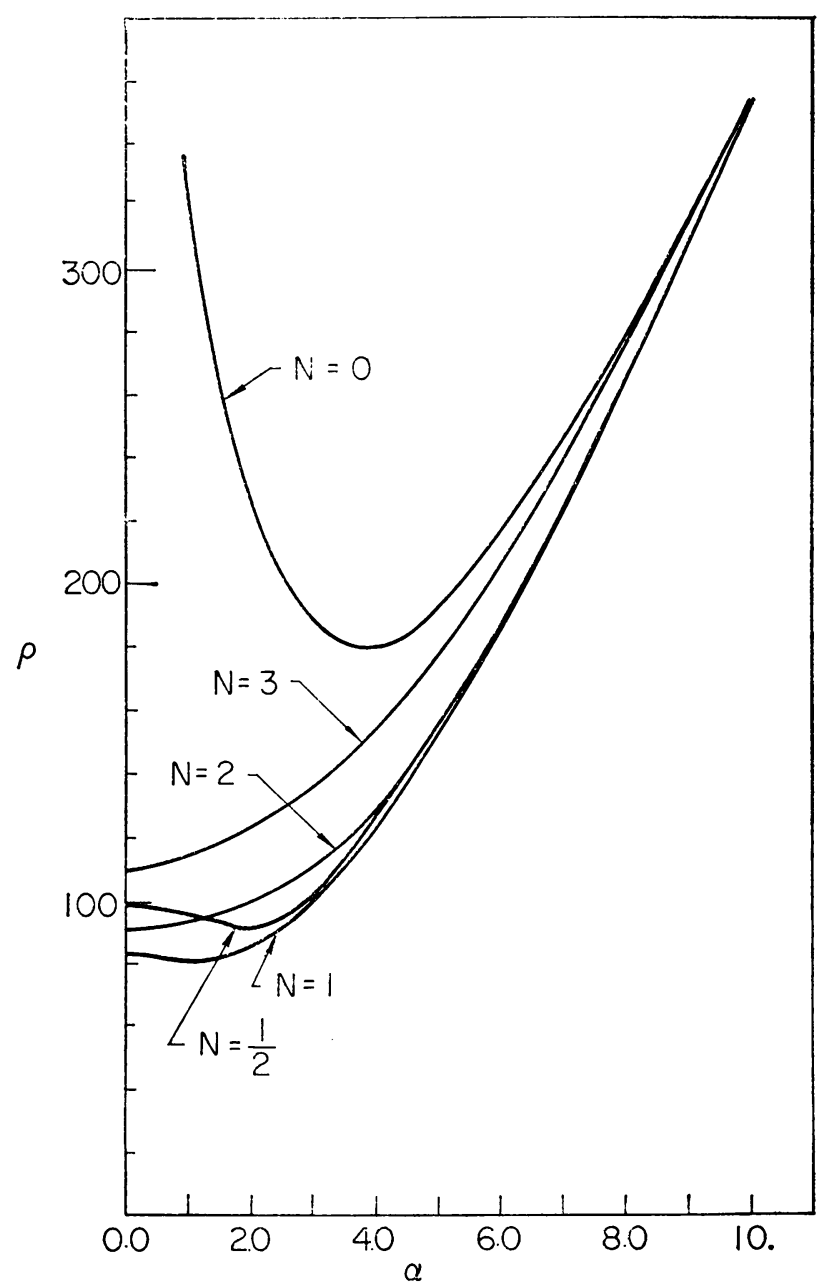

Fig. 2. Principal elgenvalues $\rho(\alpha, N)$ of Eqs. (37). The figure summarizes the results of a numerical integration of the equations. The $\rho(0, N)$ and $\rho^{(2)}(0, N)$ are obtained by exact analysis and are summarized in Tables I and II. The smallest of values $\rho(\alpha, N)$ is 81.49. For each fixed $N$ the values $\rho(\alpha, N) \rightarrow \rho(\alpha, 0)$ as $\alpha \rightarrow \infty$ (Orr's solution).

Therefore

$$
\rho \mathbf{E} \cdot \mathbf{v}=-\rho g r\left(v_{z}, 0, v_{r}\right),
$$

where $\mathbf{v}=\left(v_{r}, v_{\theta}, v_{z}\right)$ and $\mathbf{r}=(r, \theta, z)$ and $\rho=U_{M} d / \nu$. The Euler equations for this problem are (18a) and $(18 \mathrm{~b})$ with $\rho$ replaced by $\rho g(r, \eta)$. To derive these equations one need only verify that the derivation of (18a) and (18b) given in Sec. 1 may be reproduced with $\rho g(r ; \eta)$ instead of $\rho$. One can also, by following the derivation of $(37)$, arrive at the pair of equations

$$
\begin{gathered}
\rho g N^{2} f=i \alpha r D L f-\left(N^{2}+\alpha^{2} r^{2}\right) L u-2 \alpha^{2} r D u, \\
\rho g\left[\left(N^{2}+\alpha^{2} r^{2}\right) u+i \alpha r D f\right]+i \alpha \rho D g r f=L^{2} f+4 i \alpha L u,
\end{gathered}
$$


and boundary conditions

$$
f=D f=u=0 \quad \text { at } r=\eta /(1-\eta) \text { and } r=1 /(1-\eta) .
$$

The eigenvalues $\rho(\alpha, N ; \eta)$ of $(67)$ are also characterized by obvious analogues of Lemmas 1,2 , and 3. For $\alpha=0$ we are led, as before, to the system

$$
\begin{gathered}
L^{2} f-\rho g N^{2} u=0, \\
L u+\rho g f=0, \\
L=(1 / r) D(r D)-N^{2} / r^{2}, \\
f=D f=u=0 \quad \text { at } r=\eta /(1-\eta) \text { and } r=1 /(1-\eta),
\end{gathered}
$$

but since $g$ is not of one sign on $[\eta /(1-\eta), 1 /(1-\eta)]$ Lemma 4 does not hold in the annulus. The form of $g$ is such that it is not possible that the solution of the system (74) can be obtained in terms of a finite number of Bessel functions. It is possible, however, to construct a perturbation from this system and, in particular, Eqs. (65) hold with

$$
\langle a\rangle=\int_{\eta /(1-\eta)}^{1 /(1-\eta)} a r d r
$$

and $\langle u f\rangle$ and $\left\langle u^{(1)} f^{(1)}\right\rangle$ replaced by $\langle g u f\rangle$ and $\left\langle g u^{(1)} f^{(1)}\right\rangle$, respectively. The result $\rho^{(1)}$ $(0, N ; \eta)=0$ holds for all fixed $N$ and $\eta$ and the argument which leads to exclusion of a completely transverse minimizing solution for small fixed $N$, holds here also. The main difference is that for the annulus the minimizing solution over integers $N$ is such that $N \geq 2$ when $\eta \geq 10^{-4}$.

The outcome of the numerical integration of Eqs. (73) is given in Table III. Evidently the solution does reduce to the pipe flow, but even for very small radius ratios $\left(\eta \geq 10^{-4}\right)$

\section{TABLE III}

Critical Reynolds numbers for Poiseuille flow in an annular pope. The eigenvalue $\rho(0, \tilde{N} ; \eta)$ tends tonard the pipe value 82.88 as $\eta$ is decreased. For $\eta \geq 10^{-4}$ the smallest value of $\rho(\alpha, N, \eta)$ over $N$ and $\alpha, \tilde{\rho}(\tilde{a}, \tilde{N}, \eta)$ is associated with purely azimuthal solutions $(\tilde{a}=0)$. Starred values in the table mean that the search for minimum values was carried out relative to continuous $N$. The value 99.21 holds for $\eta=1$ (nearly) (plane Poiseuille flow) and has been obtained independently, and first, by Busse [10]. The integer values of $\widetilde{N}$ in this table are offered as hypotheses for experiments.

\begin{tabular}{cccc}
\hline \multicolumn{1}{c}{$\boldsymbol{\eta}$} & $\tilde{\rho}$ & $\tilde{a}$ & $\tilde{N}$ \\
0.0001 & 83.80 & 0 & $1.8^{*}$ \\
0.001 & 85.10 & 0 & $1.8^{*}$ \\
0.01 & 93.23 & 0 & $1.9^{*}$ \\
0.05 & 98.02 & 0 & 2 \\
0.20 & 100.35 & 0 & 3 \\
0.50 & 99.63 & 0 & 6 \\
0.70 & 99.43 & 0 & 12 \\
0.90 & 99.21 & 0 & 39 \\
\hline
\end{tabular}

the $N=1$ solution is not yet minimizing. In keeping with our remarks relative to the version of Eqs. (65) which hold for the annulus, $\rho\left(\alpha, 1,10^{-3}\right)$ is a local maximum when $\alpha=0$, but $\rho\left(\alpha, 2,10^{-3}\right)$ is a local minimum when $\alpha=0$ (see Fig. 3).

In Fig. 4 we have plotted the variation of the stability limit with radius ratio. The 
top curve represents the trend of the experiments [17]. The bottom curve is the energy limit. Though the values of the limits differ by an order of magnitude, their variation with the domain is remarkably similar. The linear theory, on the other hand, bears no resemblance to experiments. For the linear theory the critical Reynolds number, $U_{\max }(b-a) / \nu$, tends to $\infty$ when $\eta=0$ (pipe flow), to 10,600 when $\eta=1$ (channel flow) and the variation is monotonic in between [17].

8. Rigid rotation around the pipe axis. All of the results of the previous 7 sections hold relative to a pipe which rotates as a rigid body around its symmetry axis. For the rigidly rotating system the field $\mathrm{V}=(0, \Omega r, U(r))$ with constant angular velocity $\Omega$, is an exact solution of the Navier-Stokes equations. The constant vorticity $2 \Omega$ does not enter into the rate of strain matrix $\mathbf{E}$, into the boundary conditions on the difference motion or into the fundamental problem (2), (3) and (4). It follows that all of our results hold relative to the $\Omega$ family of rotating Poiseuille flows.

In contrast, Pedley [16] has shown that the linear limit is very sensitive to rigid rotations. In fact the result of his formal perturbation analysis (powers of $\epsilon=U_{\max } / \Omega r_{0}$ ) in the limit $\epsilon \rightarrow 0$ is exactly the solution of the energy problem when $\alpha=0$, that is

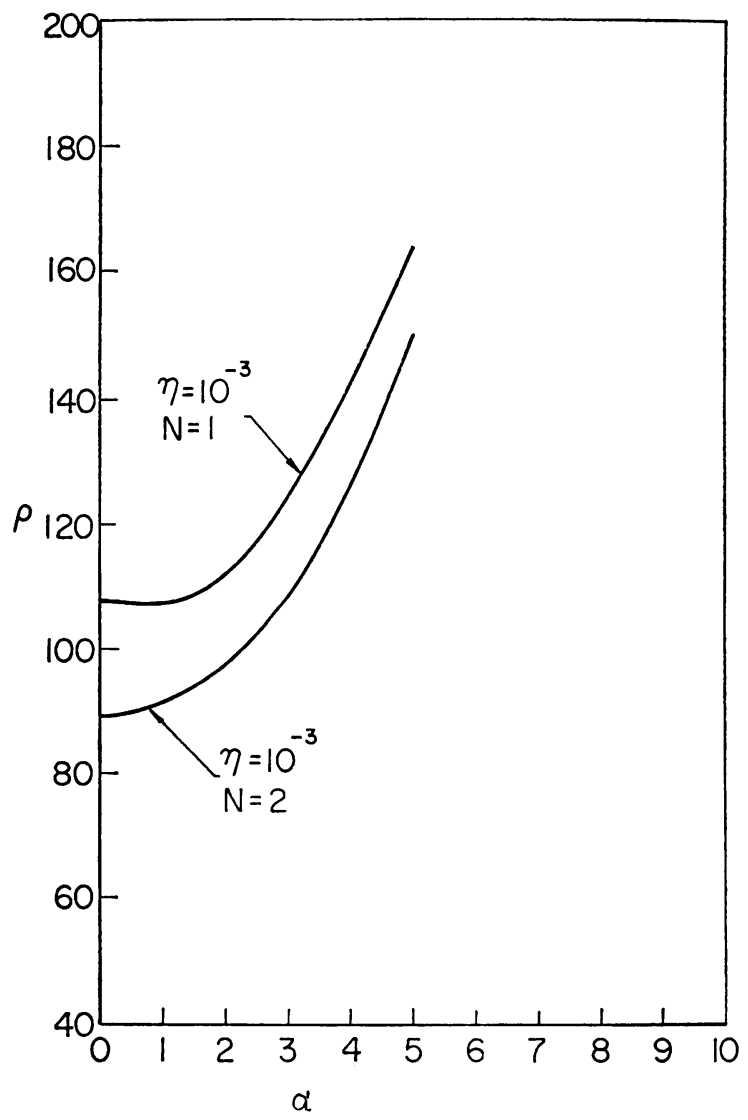

Fig. 3. Principal eigenvalues $\rho(\alpha, N ; \eta)$ for the annulus with radius ratio $\eta=10^{-3}$. The smallest eigenvalue among integer $N$ is $\rho\left(0,2 ; 10^{-3}\right)$ and it is minimum at $\alpha=0$. The eigenvalue $\rho\left(0,1 ; 10^{-3}\right)$ is a local maximum at $\alpha=0$. 
$R=82.88, N=1$ and $\alpha=0$. Pedley speaks of a flow with components $\left(0, \operatorname{sr}\left(1+0\left(\epsilon^{2}\right)\right)\right.$, $\left.1-r^{2}+0(\epsilon)\right)$, but it is not hard to show his result holds rigorously for the flow (0, $\Omega r, 1-r^{2}$ ) in the limit $\Omega \rightarrow \infty, \alpha \Omega=$ const. $^{4}$ This quite remarkable coincidence (independently derived) does not quite rule out sublinear solutions in rapidly rotating pipes (for the energy limit is 81.49 and $\alpha \neq 0$ ) but evidently does rule them out in an annular pipe $\left(\eta \geq 10^{-4}\right)$ with velocity components $\left(0, \Omega, 1-r^{2}\right)$ where the inner cylinder moves forward with velocity $1-\eta^{2}{ }^{5}$ This is the second of the such strong stability results (giving both necessary and sufficient conditions for stability) for rotating shear flows. F. Busse (private communication) has shown that the energy value $R=82.6$ is necessary as well as sufficient [5] for the stability of plane Couette flow which rotates as a rigid body in several different ways one of which involves only finite rotation rates.

9. Concluding remarks. To conclude the energy analysis of the stability (and uniqueness) of Hagen-Poiseuille flow it is necessary to determine the smallest eigenvalue $R=\rho$ of Euler's partial differential Eqs. (5) and (6). Our results fall somewhat short of this goal. We have proved that $R<82.88$. The value $R=\rho(1.07,1)=81.49$ is a numerical result but is not without a strong analytical foundation. We believe that this is the correct stability-uniqueness limit for (5) and (6).

The stability problem associated with Hagen-Poiseuille flow is in a sense a starting point for stability theory, The first systematic reports of transition are to be found in the celebrated paper of Reynolds [3] and it is in these experiments with round pipes that the "Reynolds number" criterion has its roots. For nearly a century the transition mechanism and an associated quantitative criterion have eluded exact mathematical analysis. Linear theory (as far as its result is known) gives stability at all Reynolds numbers even to nonaxisymmetric disturbances (Salwen and Grosch [13]; Lessen, Sadler and Liu [14], The linear theory is not totally at variance with experiment (Leite [15] and [14]). If care is taken to keep extraneous disturbances small enough the transition Reynolds number can be made very large. Presumably, truly infinitesimal disturbances would never amplify. Reynolds [3] himself had no confidence in the ability of linear theory to explain his transition observations and notes that the abruptness of the transition "at once suggested the idea that the condition might be one of instability for disturbances of a certain magnitude, and stable for smaller disturbances."

The linear theory fails also for the natural instabilities of the Poiseuille flows in annular spaces. This is abundantly clear when $\eta=0$ (pipes) but it is also true when $\eta=1$ (channels) and is increasingly true for the small $\eta$ in between. It is true that Lin's [21] number 10,600 is relatively closer to the experimental 2000 than the 99.20 of energy theory. Neither value accurately mirrors experiments and this is not surprising. The linear limit is taken on in a class of disturbances narrower than true solutions and it can be radical (but not conservative) relative to experiments. The energy limit is taken on in a class of disturbances wider than true solutions and it can be conservative (but not radical) relative to experiments.

One cannot know, a priori, whether energy or linear solutions will more faithfully reflect certain features of the nonlinear hydrodynamics. Figure 4 suggests that the energy

${ }^{4}$ To do this we show that Euler's equation [5] and the linear perturbation equations (written in the rigid body coordinates) differ at fixed $R$ when $\alpha \rightarrow 0$ and $\alpha \Omega=$ const. by the gradient of scalar field. The linear eigenvalue $R(\Omega)$ tends to 82.88 as $\Omega \rightarrow \infty$.

${ }^{6}$ To establish this one needs to show that the lowest of the values $\rho(\alpha, N ; \eta)$ occurs in the set $\alpha=0$. Numerical analysis shows this is true when $\eta \geq 10^{-4}$. 


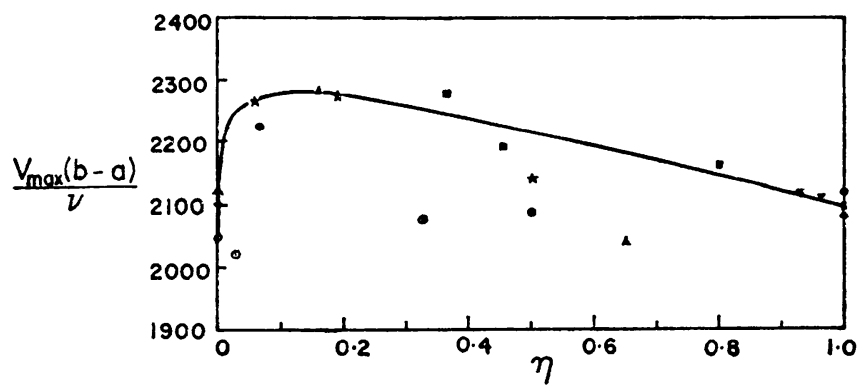

(a)

(a) Experimental values giving the transition point for the breakdown of Poiseuille flow in annuli. The points were compiled by $R$. Hanks [16] and the relevant experiments are listed in his paper. The points with white interiors are presumably less reliable than the black ones. The curve is an interpolation formula devised by Hanks. We have left his curve on the figure to show the trend of the experimental results.

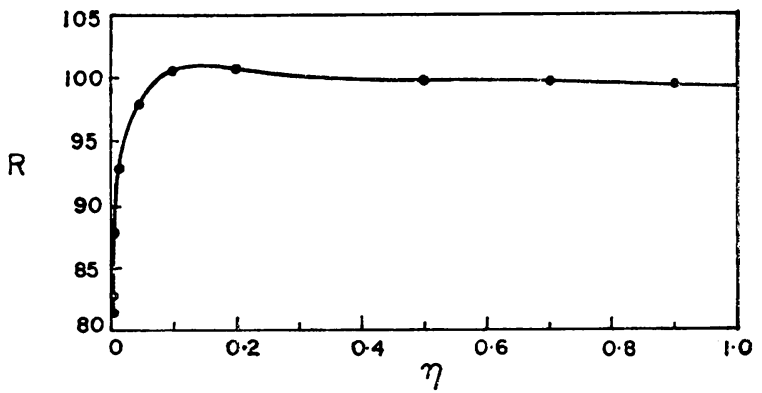

(b)

(b) Energy stability limit as a function of the radius ratio. The points on the curve are stability limits (numerical). The stability limit occurs among integers $N$ and the true curve has discontinuous first derivatives. The curve shown is a smooth representation of the true curve. The last five entries of Table III are stability limits and for these $R=\tilde{\rho}$. For $\eta=0, R=\rho(1.07,1)=81.49$. For $\eta=10^{-4}, R=$ $\rho(0,2)=88.75$. For $\eta=0.01, R=\rho(0,2)=93.31$. For $\eta=1, R=99.20$.

FIG. 4.

limit may retain more of the physics than the linear limit. The variation of the linear limit with $\eta$ seems to have nothing in common with experiments [18]. On the otherhand the experimental and energy limits are alike with regard to their variation with $\eta$. This resemblance may, however, be fortuitous and a critical experimental test of the relevance of energy eigenfunctions can be found in the "predicted" dramatic variation of the azimuthal periodicity $(N(\eta))$ with $\eta$ (Table III).

The factor of about 20 which separates the energy and experimental limits is not to be denied: neither should it be overemphasized. In the "fast" rotation limit even this difference must vanish, nearly.

Ordinarily, however, one cannot expect detailed agreement between the result of energy analysis and the true nonlinear hydrodynamics. For this to hold, it would be sufficient that the Euler problem for the energy functional and the linearized hydrodynamic stability problem coincide (as for the Bénard problem and some rotating shear flows [5]). For parallel motions (with $\Omega=0$ ) we have no such coincidence and the solutions of the Euler equations quite clearly cannot reduce the linearized stability problem to an 
identity. Moreover, when there is not such coincidence, nonenergy integral constraints (vorticity integrals, moments of various kinds, etc.) on the hydrodynamic solutions will not hold relative to the solutions of Euler's equations. For example, the extremal solution for Poiseuille flow in annuli (for $\eta \geq 10^{-4}$ ) are found in a class of two dimensional solutions which do not include possible amplified or neutral solutions of the hydrodynamic problem [19].

What then is the physical content of solutions of the Euler problem? Of all the kinematically possible difference motions (these are solenoidal, and satisfy the boundary conditions) the ones which extremalize the eigenvalues of Euler's equations are also most efficient in converting the energy of the basic motion into energy of the difference motion. Such energetically efficient fields are not always dynamically possible. But the energetically efficient component of true hydrodynamic fields are quite possibly most relevant for instability. It is in this context that we note that the three central experimentally observed features of the most destabilizing disturbance for pipe flow [2], the spiral mode, the first mode $(N=1)$ azimuthal periodicity, and the finite amplitude of the disturbance, as well as the domain dependence of the experimental transition limits in annuli, are all inevitable outcomes of energy analysis without approximations or appeal to physical intuition.

This work was supported under NSF Grant GK-1838. A part of this work constitutes a part of doctoral dissertation of S. Carmi. We wish to thank Mr. R. Hotchkiss of the Computer Center of the University of Minnesota for writing the Bessel function subroutine used in our calculation. To Mr. W. Hung we are indebted for checking some of the numerical calculations.

Appendix. Frobenius series as starting values for numerical solutions of differential equations which have regular singularities at the origin. We illustrate the method first by application to the real system (59) and then to the complex system (41a).

(a) Real-sixth order system. Consider the boundary value problem (59).

$$
\begin{gathered}
L^{3} f=-N^{2} \rho^{2} f, \\
f=D f=L^{2} f=0 \text { at } r=1, \\
f \text { bounded at } r=0 .
\end{gathered}
$$

We expect three linearly independent solutions of this problem and seek them as Frobenius series

$$
f(r)=\sum_{l=0}^{\infty} c_{l} r^{\beta+l}
$$

The coefficients $c_{l}$ are found recursively as the coefficients of $r^{\beta+l}$ in the series which arises from forming $L^{3} f=-N^{2} \rho^{2} f$ with (1A)

where

$$
L^{3} f=\sum_{l=0}^{\infty} d_{l} c_{l} r^{\beta+l-6},
$$

$$
d_{l}=\prod_{\nu=0}^{2}\left\{(\beta+l-2 \nu-1)(\beta+l-2 \nu)+(\beta+l-2 \nu)-N^{2}\right\} .
$$

The values of $\beta$ for which this procedure can be carried out are determined by the index equation

There are six roots of $(4 \mathrm{~A})$.

$$
d_{0}=0 \text {. }
$$




$$
\left(\beta_{1}, \beta_{2}, \beta_{3}, \beta_{4}, \beta_{5}, \beta_{6}\right)=(N,-N, N+2,-N+2, N+4,-N+4) .
$$

Consider the largest roots $\left(\beta_{1}, \beta_{3}, \beta_{5}\right)$. These three solutions are found to be linearly independent (see Ince [20]) and are regular at the origin. When $N$ is larger than 4 the solutions corresponding to $\beta_{2}, \beta_{4}, \beta_{6}$ are singular at the origin. When $1 \leq N \leq 4$ solutions belonging to $\beta_{4}$ or $\beta_{6}$ may be regular but they are linearly dependent on other solutions and hence lead to independent solutions with logarithmic singularities. Hence for all $N>0$ only the solutions corresponding to $\beta_{1}, \beta_{3}$ and $\beta_{5}$ satisfy the required conditions. Then,

$$
f_{1}=r^{N}+0\left(r^{N+6}\right), \quad f_{2}=r^{N+2}+0\left(r^{N+8}\right), \quad f_{3}=r^{N+4}+0\left(r^{N+10}\right),
$$

are the leading terms of three independent solutions of $(59)^{6}$. A linear combination of these three solutions is a solution provided that this combination satisfies the boundary conditions at $r=1$, that is,

$$
\Delta(\rho, N)=\left|\begin{array}{ccc}
f_{1}(1) & f_{2}(1) & f_{3}(1) \\
D f_{1}(1) & D f_{2}(1) & D f_{3}(1) \\
L^{2} f_{1}(1) & L^{2} f_{2}(1) & L^{2} f_{3}(1)
\end{array}\right|=0 .
$$

The nine elements of $\Delta$ may be provided by numerical integration of (59) using the Runge-Kutta-Gill method. Each solution $f_{1}, f_{2}$ and $f_{3}$ separately satisfies the differential equations but only a linear combination can ordinarily satisfy the boundary conditions of (59). The differential equation can be integrated from starting values $d^{i} f_{i}\left(\epsilon_{0}\right) / d r^{i}$, $j=0-5, i=1,2,3$ which are obtained from (5A) with $r=\epsilon_{0}$. A value of $\rho$ must also be guessed to provide elements for $\Delta$. An iterative procedure for the least value of $\rho$ (the smallest zero of $(6 \mathrm{~A})$ ) is then employed and a value $\rho\left(\epsilon_{0}\right)$ determined. This procedure is repeated for a sequence of values $0<r=\epsilon_{i} \rightarrow 0$ and new values of $\rho\left(\epsilon_{i}\right)$ are determined. Convergence is determined according to $\rho\left(\epsilon_{i+1}\right)-\rho\left(\epsilon_{i}\right)<\delta$ for a preassigned $\delta$ for all $i \geq J \geq 0$. The criterion is not exact for one cannot set $\epsilon=0$. The error in the starting values is of the order of the next term neglected in the Frobenius series (5A). In practice, convergence to four significant figures is very rapid. The values of Table $I$ have been checked using this procedure and are in perfect agreement with the exact Bessel function solution.

(b) Complex fourth-order system. For Orr's problem (41a) we cannot provide explicit solutions and must rely on the numerical integration. We first rewrite (41a) and $(37 \mathrm{c})$.

$$
\begin{gathered}
D^{2} f=2 i \alpha \rho r D f, \\
f=D f=0 \text { at } \quad r=1, \\
f \text { bounded at } \quad r=0,
\end{gathered}
$$

where $\mathscr{D}=D^{2}-(1 / r) D-\alpha^{2}$. Frobenius series (1A) with complex coefficients $c_{l}$ are next substituted in (7A). The coefficient of each power of $r$ is equated to zero. The lowest order term $\left(r^{l+\beta-4}\right)$ is found in the expression for 


$$
\begin{aligned}
\mathscr{D}^{2} f=\sum[(\beta+l-2)(\beta+ & l-3) \\
& -(\beta+l-2)][(\beta+l)(\beta+l-1)-(\beta+l)] c_{l} r^{l+\beta-4} \\
- & 2 \sum \alpha^{2}[(\beta+l)(\beta+l-1)-(\beta+l)] c_{l} r^{\beta+l-2}+\sum \alpha^{4} c_{l} r^{\beta+l} .
\end{aligned}
$$

The coefficient of this term with $l=0$ yields the index equation

$$
[(\beta-2)(\beta-3)-(\beta-2)][\beta(\beta-1)-\beta]=\beta(\beta-2)^{2}(\beta-4)=0 .
$$

Of the four roots $\left(\beta_{1}, \beta_{2}, \beta_{3}, \beta_{4}\right)=(0,2,2,4)$ only $\beta_{3}$ and $\beta_{4}$ are independent (see Ince [20]). Two other solutions may be formed but these have logarithmic singularities at the origin and must be discarded.

$A_{1} r^{2}$ and $A_{2} r^{4}$ where $A_{1}$ and $A_{2}$ are complex are the leading terms of two independent solutions of $(7 \mathrm{~A})$ and provide starting values for the numerical integration. To satisfy the boundary conditions we must have

$$
\Delta(\rho, \alpha)=\left|\begin{array}{cc}
f_{1}(1) & f_{2}(1) \\
D f_{1}(1) & D f_{2}(1)
\end{array}\right|=0,
$$

In practice we use an equivalent system of equations for the integration. We set $f=\varphi+i \Psi$ and

$$
\varphi=\sum_{i=1}^{4} A_{i} \varphi_{i}, \quad \Psi=\sum_{i=1}^{4} A_{i} \Psi_{i},
$$

where the pair $\left(\varphi_{i}, \Psi_{i}\right)$ satisfies the differential equations

$$
D^{2} \varphi_{i}=-2 \alpha \rho r D \Psi_{i}, \quad D^{2} \Psi_{i}=2 \alpha \rho r D \varphi_{i},
$$

but not the boundary conditions.

The boundary conditions

$$
\begin{gathered}
\varphi(1)=\Psi(1)=D \varphi(1)=D \Psi(1)=0, \\
\varphi, \Psi \text { bounded at } r=0,
\end{gathered}
$$

are satisfied if

$$
\Delta(\rho, \alpha)=\left|\begin{array}{cccc}
\varphi_{1}(1) & \varphi_{2}(1) & \varphi_{3}(1) & \varphi_{4}(1) \\
D \varphi_{1}(1) & D \varphi_{2}(1) & D \varphi_{3}(1) & D \varphi_{4}(1) \\
\Psi_{1}(1) & \Psi_{2}(1) & \Psi_{3}(1) & \Psi_{4}(1) \\
D \Psi_{1}(1) & D \Psi_{2}(1) & D \Psi_{3}(1) & D \Psi_{4}(1)
\end{array}\right|=0
$$

The elements of $\Delta(\rho, \alpha)$ are obtained by the direct integration of $(9 \mathrm{~A})$ with the starting values

$$
\begin{array}{lll}
\varphi_{1}=r^{2}, & \varphi_{2}=\varphi_{3}=\varphi_{4}=0, & \Psi=0, \\
\varphi_{2}=r^{4}, & \varphi_{1}=\varphi_{3}=\varphi_{4}=0, & \Psi=0, \\
\Psi_{3}=r^{2}, & \Psi_{1}=\Psi_{2}=\Psi_{4}=0, & \varphi=0, \\
\Psi_{4}=r^{4}, & \Psi_{1}=\Psi_{2}=\Psi_{3}=0, & \varphi=0,
\end{array}
$$

again using the Runge-Kutta-Gill method. 
We obtain the least root $\rho$ of (11A) for a fixed $\alpha$ and $\epsilon_{i}$ by an iterative procedure similar to that described under (a). Then we seek $\operatorname{Min}_{\alpha} \rho(\alpha)$. The result is $\operatorname{Min}_{\alpha} \rho(\alpha)=$ 180.6, $\alpha=3.6$. This compares with $\operatorname{Min} \rho(\alpha) \cong 180$., $\alpha \cong 3.7$, which has been calculated by hand by Orr.

\section{REFERENCES}

[1] W. McF. Orr, The stability or instability of steady motions of a liquid, Part II: A viscous liquid, Proc. Roy. Irish. Acad. Sect. (A) 27, 69-138 (1907)

[2] J. Fox, M. Lessen and W. Bhat, Experimental investigation of the stability of Hagen-Poiseuille flow, Phys. Fluids. 11, 1 (1968)

[3] O. Reynolds, On the dynamical theory of incompressible viscous fluids and the determination of the criterion, Philos. Trans. Roy. Soc. London Ser. A 186, 123-164 (1895)

[4] J. Serrin, On the stability of viscous fluid motions, Arch. Rational Mech. Anal. (1) 3, 1-13 (1959)

[5] D. D. Joseph, Nonlinear stability of the Boussinesg equations by the method of energy, Arch. Rational Mech. Anal. (3) 22, 163-184 (1966)

[6] F. Reisz, and B. Sz.-Nagy, Lecons d'analyse fonctionnelle, Chap. VI, Akad. Kiado, Budapest, 1925

[7] W. Velte, Über ein Stabilitätskriterium der Hydrodynamik, Arch. Rational Mech. Anal. 9, 9-20 (1962)

[8] Von Klaus Kirchgässner, Die Instabilität der Stromüng zwischen zwer rotierenden Zylindern gegenüber Taylor-Wirbeln für beliebige Spaltbreiten, Z. Angew. Math. Phys. 12, 14-30 (1961)

[9] R. Jentzsch, Über Integralgleichungen mit positivem Kern, J. Math. 141, 235 (1912)

[10] F. Busse, Bounds on turbulent transport of mass and momentum, Z. Angew. Math. Phys.

[11] L. Y. Hung, Stability of Couette flow by the method of energy, M.S. thesis, Department of Aeronautics and Engineering Mechanics, University of Minnesota (1968)

[12] T. Kato, Perturbation theory for linear operators, Springer-Verlag, New York, 1966

[13] H. Salwen and E. Grosch, Stability of Poiseuille flow in a circular pipe, Bull. Amer. Phys. Soc. (Abstract) 13, 814 (1968)

[14] M. Lessen, S. Sadler and T. Liu, Stability of pipe Poiseuille flow, Phys. Fluids. 11, 1404-1409 (1968)

[15] R. J. Leite, An experimental investigation of the stability of Poiseuille flow, J. Fluid Mech. 5, 81 (1959)

[16] T. J. Pedley, On the instability of viscous flow in a rapidly rotating pipe, J. Fluid Mech. (in press)

[17] R. Hanks, The laminar-turbulent transition for flow in pipe, concentric annuli, and parallel plates, A. I. Ch. E. J. 9 (1963), 45-48

[18] J. Mott and D. Joseph, Stability of parallel flow between concentric annuli, Phys. Fluids (1968), (to appear)

[19] D. D. Joseph and L. N. Tao, Transverse velocity components in fully-developed flows, J. Appl. Math. Mech. 30, 147-148 (1963)

[20] E. L. Ince, Ordinary differential equations, Dover, New York, 1956, p. 405

[21] C. C. Lin, On the stability of two-dimensional parallel flow, Quart. Appl. Math. 3, 277 (1966) 